\title{
Association of C3435T (rs1045642) Polymorphism of the MDR 1 Gene with the Increased Risk of Colorectal Cancer in Russian Females from Central Russia
}

\author{
A. S. Moskalev ${ }^{a, b}$, E. M. Barysheva ${ }^{c}$, V. O. Soldatov ${ }^{c, d}$, O. G. Frolova ${ }^{e}$, O. V. Bobyntseva ${ }^{a, c}$, \\ T. A. Samgina $f$, M. I. Churnosov ${ }^{g}$, V. P. Ivanov ${ }^{c}$, A. V. Polonikov, ${ }^{a}$, and O. Yu. Bushueva ${ }^{a, c}$, * \\ ${ }^{a}$ Research Institute of Genetic and Molecular Epidemiology, Kursk State Medical University, Kursk, 305004 Russia \\ ${ }^{b}$ Kursk Regional Clinical Oncology Dispensary, Kursk oblast, farmstead of Kislino, 305524 Russia \\ ${ }^{c}$ Department of Biology, Medical Genetics, and Ecology, Kursk State Medical University, Kursk, 305004 Russia \\ ${ }^{d}$ Department of Pharmacology and Clinical Pharmacology, Belgorod National Research University, Belgorod, 308015 Russia \\ ${ }^{e}$ Department of Oncology, Kursk State Medical University, Kursk, 305004 Russia \\ ${ }^{f}$ Department of Surgical Diseases No. 2, Kursk State Medical University, Kursk, 305004 Russia \\ ${ }^{g}$ Department of Biomedical Disciplines, Belgorod National Research University, Belgorod, 308015 Russia \\ *e-mail: olga.bushueva@inbox.ru \\ Received January 12, 2019; revised March 12, 2019; accepted April 9, 2019
}

\begin{abstract}
The objective of this study was to examine associations of single nucleotide polymorphisms, rs1045642 within the MDR1 gene and rs1799930 within the NAT2 gene, with the risk of colorectal cancer (CRC) in the population of Central Russia. DNA specimens were obtained from 178 patients with CRC ( 87 males and 91 females) and 327 age-matched healthy controls (179 males and 148 females). Genotyping was performed using real-time PCR. Association of the studied SNPs with the risk of CRC was evaluated using logistic regression analysis. It was demonstrated that MDR1 rs 1045642 polymorphism was associated with the increased risk of CRC after correction for gender, age, and smoking $(O R=1.41,95 \% C I=1.09-$ $1.83 ; P=0.008)$. A gender-stratified analysis showed that $M D R 1$ rs 1045642 was associated with the increased risk of CRC only in females $(O R=1.62,95 \% C I=1.11-2.35 ; P=0.01)$. In males, no association between MDR1 rs1045642 and CRC was found. Association between MDR1 rs1045642 and the increased risk of colorectal cancer in Russian females from Central Russia was revealed.
\end{abstract}

Keywords: colorectal cancer, hereditary predisposition, MDR1, NAT2, rs1045642, rs1799930

DOI: $10.1134 / \mathrm{S} 1022795419120093$

\section{INTRODUCTION}

Colorectal cancer (CRC), including colon and rectal cancer, is one of the most common cancers worldwide [1]. Previous epidemiological studies have shown that genetic susceptibility factors together with environmental factors (such as smoking, nutrition, bacterial toxins, etc.) can increase the risk of CRC [2]. However, the exact mechanisms underlying the development of this malignant tumor of the digestive system remain unclear.

Arylamine N-acetyltransferase 2 (NAT2) is an enzyme involved in the Phase II of xenobiotic biotransformation. It catalyzes the metabolism of environmental carcinogens (aromatic hydrocarbons, heterocyclic amines, and hydrazines), as well as some polar compounds (such as methyl and acetyl) [3]. NAT2 is most highly expressed in the liver and intestinal mucosa. The gene encoding NAT2 is polymorphic. The $\mathrm{G}>$ A substitution at the position 590 of
NAT2 (rs1799930) determines the acetylator phenotype, which is subdivided into rapid and slow ones. The acetylator status, in turn, can affect the metabolism of certain carcinogens, the activation/inactivation of which may be at risk of CRC. Analyses of associations between the NAT2 acetylator phenotypes and CRC showed conflicting results. For instance, ProbstHensch et al. [4] found an inverse correlation between the NAT2 rapid acetylator genotype and colorectal adenoma in black Americans; however, in white Americans, this genotype was associated with the increased risk of this disease. Other studies have also shown that the rapid NAT2 genotype played an important role in susceptibility to colorectal cancer [5]. At the same time, previous meta-analysis showed a lack of association between NAT2 rs1799930 and CRC [6].

The multidrug resistance 1 gene (MDR1, also called the ATP-binding cassette transporter, subfamily 
$\mathrm{B}$, member $1(A B C B 1))$ encodes transmembrane glycoprotein, $\mathrm{P}$-glycoprotein (P-gp), participating in the Phase III of xenobiotic biotransformation [7]. C3435T (rs1045642), one of the most studied SNPs in the MDR1 gene, affects the $\mathrm{P}$-gp functional properties [8]. In 2005, Kurzawski et al. [9] were analyzed, for the first time, an association between MDR1 C3435T and the development of CRC in the Polish population. It was demonstrated that MDR1 3435TT genotype was associated with a 2.7 -fold higher risk of colon cancer. Then, the number of studies on the association of this polymorphism with the development of CRC increased considerably with most of them performed on Asian populations. However, the results were contradictory [10-12]. For instance, Komoto C. et al. [11] revealed the effect of MDR1 rs1045642 on the development of CRC in the Japanese population [11]. At the same time, in two other studies performed in Korea, no associations between rs 1045642 MDR 1 and CRC were found [10, 12]. These contradictions can be 1 explaned by the small sample sizes of the studied cohorts and low statistical power of the studies. A recent meta-analysis showed a lack of association between MDR1 C3435T and the risk of colorectal cancer in Europeans [13]. Another recent meta-analysis showed the association between MDR1 C3435T polymorphism and a decreased risk of CRC in Asians [14].

Associations between NAT2 rs1799930 and MDR1 rs 1045642 polymorphisms and the risk of colorectal cancer in the population of Central Russia were not 2 studed yet.

The objective of this study was to analyze the associations between NAT2 rs1799930 and MDR1 rs 1045642 polymorphisms and the risk of colorectal cancer in the population from Central Russia.

\section{MATERIALS AND METHODS}

A total of 505 unrelated Russian individuals from Kursk region was examined; written informed consent was obtained from all participants prior to entering the study. The study included $178 \mathrm{CRC}$ patients ( 87 males and 91 females) who underwent inpatient treatment at the Kursk Regional Clinical Oncology Center between 2013 and 2017 [15]. The control group consisted of 327 healthy volunteers (179 males and 148 females) without a history of chronic diseases. Patients were included in the study group only after verification of final diagnosis of the disease, confirmed by clinical, laboratory and instrumental methods. The study was approved by the Regional Ethics Committee of Kursk State Medical University.

The mean age of CRC patients was $66.92 \pm 9.27$ years; the mean age of the control group individuals was slightly higher $-68.71 \pm 8.44$ years $(P=0.02)$. The groups (CRC patients and controls) were frequency-matched according to gender $(P>0.05)$. Venous blood samples were obtained from all individ- uals. Genomic DNA was isolated from peripheral blood by the standard phenol-chloroform extraction method. Genotyping of NAT2 rs1799930 and MDR1 rs 1045642 polymorphisms was performed by realtime PCR using allelic discrimination assay with TaqMan probes on a CFX96 amplifier (Bio-Rad, USA). Regenotyping of $10 \%$ of the studied samples taken randomly and in the absence of information on the status of the disease showed $100 \%$ reproducibility of the original results.

Associations of alleles with the risk of CRC were performed using logistic regression analysis. To assess association of genotypes with the disease, the odds ratio $(O R)$ and $95 \%$ confidence interval $(C I)$ values calculated for the log-additive regression model were used. All calculations were performed adjusted for gender, age, and smoking in the SNPStats software program, available online (https://www.snpstats.net/start.htm). A value of $P \leq 0.05$ was taken as statistically significant.

\section{RESULTS AND DISCUSSION}

The genotype frequency distributions of the studied SNPs in the patient and control groups were in Hardy-Weinberg equilibrium $(P>0.05)$. We were unable to genotype $M D R 1$ rs 1045642 polymorphism in one DNA specimen from the CRC patient group, as well as in one DNA specimen from the control group. The results of the analysis of associations of the studied polymorphisms with SRS risk are presented in Table 1. According to the results, NAT2 rs1799930 was not associated with the risk of colorectal cancer; at the same time, MDR1 rs1045642 was associated with the risk of CRC even after adjustment for gender, age, and smoking habit (Table 1).

Taking into consideration the sexual dimorphism of associations of the genetic markers with multifactorial diseases, at the next step we performed genderstratified analysis of rs 1045642 in the MDR1 gene with the risk of colorectal cancer. In males, no associations between MDR1 rs 1045642 and the risk of CRC were detected. At the same time, in females, MDRI rs 1045642 polymorphism was associated with the risk of CRC, taking into account adjustment for gender, age, and smoking habit (Table 2).

A number of studies accumulated over the last years provide evidence on the important role of genetic factors in the pathogenesis of CRC [9-11].

The MDRl gene, which encodes the ATP-binding cassette transporter, $\mathrm{P}-\mathrm{gp}$, is mapped to chromosomal region $7 \mathrm{q} 21.12$. It contains 29 exons and encodes a protein containing 1280 amino acids [7]. To date, more than 50 single nucleotide polymorphisms have been identified in the MDR1 gene. A recent study showed that synonymous substitution (C3435T) was one of the most functionally important polymorphisms in the MDR1 gene, which changed the conformation of the encoded P-gp and affected the timing of 
Table 1. Analysis of associations of NAT2 rs1799930 and MDR1 rs1045642 with the risk of colorectal cancer

\begin{tabular}{|c|c|c|c|c|c|}
\hline \multicolumn{6}{|c|}{ Analysis of associations of $N A T 2$ rs 1799930 with the risk of CRC, combined groups } \\
\hline & Minor allele/genotypes & $\begin{array}{l}\text { Patients } \\
(N=178)\end{array}$ & Control $(N=327)$ & $P^{1}$ & $\begin{array}{c}\text { OR } \\
(95 \% C I)^{2}\end{array}$ \\
\hline $\begin{array}{l}\text { Minor allele frequency } \\
\text { Genotype frequencies, } \\
N(\%)\end{array}$ & $\begin{array}{l}A \\
G G \\
G A \\
A A\end{array}$ & $\begin{array}{l}0.272 \\
93(52.2 \%) \\
73(41 \%) \\
12(6.7 \%)\end{array}$ & $\begin{array}{c}0.291 \\
167(51.1 \%) \\
130(39.8 \%) \\
30(9.2 \%)\end{array}$ & 0.59 & $\begin{array}{c}0.92 \\
(0.69-1.22) \\
0.93 \\
(0.70-1.24)\end{array}$ \\
\hline \multicolumn{6}{|c|}{ Analysis of associations of $M D R 1$ rs 1045642 with the risk of CRC, combined groups } \\
\hline & Minor allele/genotypes & $\begin{array}{l}\text { Patients } \\
(N=177)\end{array}$ & Control $(N=326)$ & $P^{1}$ & $\begin{array}{c}\text { OR } \\
(95 \% C I)^{2}\end{array}$ \\
\hline Minor allele frequency & & 0.531 & 0.440 & 0.01* & $\begin{array}{c}1.44 \\
(1.11-1.87)\end{array}$ \\
\hline $\begin{array}{l}\text { Genotype frequencies, } \\
N(\%)\end{array}$ & $\begin{array}{l}C C \\
C T \\
T T\end{array}$ & $\begin{array}{l}41(23.2 \%) \\
84(47.5 \%) \\
52(29.4 \%)\end{array}$ & $\begin{array}{r}106(32.5 \%) \\
153(46.9 \%) \\
67(20.6 \%)\end{array}$ & $0.008^{* !}$ & $\begin{array}{c}1.41^{!} \\
(1.09-1.83)^{!}\end{array}$ \\
\hline
\end{tabular}

${ }^{1} P$ value with adjustment for gender, age, and smoking habit. ${ }^{2}$ Odds ratio and $95 \%$ confidence interval. ${ }^{3}$ Akaike information criterion. * Statistically significant differences between groups are demonstrated. ' Indices calculated for the log-additive regression model with adjustment for gender, age, and smoking habit.

Table 2. The influence of gender on associations of $M D R 1$ rs1045642 with the risk of colorectal cancer

\begin{tabular}{|c|c|c|c|c|c|}
\hline \multicolumn{6}{|c|}{ Analysis of associations of $M D R 1$ rs 1045642 with the risk of CRC, males } \\
\hline & Minor allele/genotypes & $\begin{array}{l}\text { Patients } \\
(N=86)\end{array}$ & $\begin{array}{l}\text { Control } \\
(N=180)\end{array}$ & $P^{1}$ & $\begin{array}{c}\text { OR } \\
(95 \% C I)^{2}\end{array}$ \\
\hline Minor allele frequency & $T$ & 0.500 & 0.444 & 0.27 & $\begin{array}{c}1.25 \\
(0.87-1.80)\end{array}$ \\
\hline \multirow{3}{*}{$\begin{array}{l}\text { Genotype frequencies, } \\
N(\%)\end{array}$} & $C C$ & $20(23.3 \%)$ & $58(32.2 \%)$ & & 1.25 \\
\hline & $C T$ & $46(53.5 \%)$ & $84(46.7 \%)$ & 0.23 & $(0.87-1.79)$ \\
\hline & $T T$ & $20(23.3 \%)$ & $38(21.1 \%)$ & & \\
\hline \multicolumn{6}{|c|}{ Analysis of associations of $M D R 1$ rs10 45642 with the risk of CRC, females } \\
\hline & Minor allele/genotypes & $\begin{array}{l}\text { Patients } \\
(N=91)\end{array}$ & $\begin{array}{l}\text { Control } \\
(N=146)\end{array}$ & $P^{1}$ & $\begin{array}{c}\text { OR } \\
(95 \% C I)^{2}\end{array}$ \\
\hline Minor allele frequency & $T$ & 0.560 & 0.435 & $0.01 *$ & $\begin{array}{c}1.65 \\
(1.14-2.40)\end{array}$ \\
\hline \multirow{3}{*}{$\begin{array}{l}\text { Genotype frequencies, } \\
N(\%)\end{array}$} & $C C$ & $21(23.1 \%)$ & $48(32.9 \%)$ & \multirow{3}{*}{$0.01 * !$} & \multirow{3}{*}{$\begin{array}{c}1.62 ! \\
(1.11-2.35) !\end{array}$} \\
\hline & $C T$ & $38(41.8 \%)$ & $69(47.3 \%)$ & & \\
\hline & $T T$ & $32(35.2 \%)$ & $29(19.9 \%)$ & & \\
\hline
\end{tabular}

${ }^{1} P$ value with adjustment for gender, age, and smoking habit. ${ }^{2}$ Odds ratio and $95 \%$ confidence interval. ${ }^{3}$ Akaike information criterion. ${ }^{*}$ Statistically significant differences between groups are demonstrated. ${ }^{!}$Indices calculated for the log-additive regression model with adjustment for gender, age, and smoking habit.

cotranslational folding and introduction of P-gp into the membrane, thereby changing the structure of substrate and inhibitor interaction sites [8]. In addition, Hoffmeyer et al. [16] showed a considerable decrease in the level of MDR1 protein in duodenal tissues of homozygous 3435TT carriers. Thus, rs1045642 polymorphism affects both the MDR1 gene expression and P-glycoprotein activity. 
Data on association of MDR $1 \mathrm{C} 3435 \mathrm{~T}$ with the risk of CRC are contradictory. In some studies, a pronounced association between MDR1 C3435T and susceptibility to CRC was demonstrated $[17,18]$. In other studies, association was not confirmed [10-12, 19]. To study the association of the MDR1 gene with the risk of CRC, a number of meta-analyses were performed. For example, according to the results of a meta-analysis which included ten studies of European populations, no association between MDR1 C3435T polymorphism and colorectal cancer was detected [13]. However, He T. et al. [20] showed that combined haplotypes of several MDR1 SNPs, including rs1045642, could be a reliable marker to determine the susceptibility to CRC in Europeans, while MDR1 rs1045642 polymorphism was highly associated with the risk of $\mathrm{CRC}$ in Asians. Another large meta-analysis based on 39 studies of different forms of cancer showed that carriage of the MDR1 3435TT genotype was associated with an increased risk of different forms of cancer in European populations [21].

It is known that the relative contribution of genetic markers to susceptibility to CRC may vary depending on the ethnic group. Moreover, a recent study showed a considerable difference in the prevalence of the MDR1 3435TT genotype among healthy Asians (27.8\%) and Europeans (49.4\%) [22]. Thus, the relative contribution of MDR1 C3435T polymorphism to the development of CRC may vary in different populations, and the role of MDR 1 rs 1045642 in colon carcinogenesis remains unclear.

The present study involved 178 CRC patients and 327 individuals from the control group. This is the first study of association between MDR 1 C3435T polymorphism and susceptibility to CRC in the Russian population. The data obtained showed that MDR1 rs1045642 was associated with the increased risk of CRC in the population of Central Russia.

The ATP-binding cassette transporter B1 (ABCB1, P-glycoprotein, multidrug resistance protein 1 (MDR1)) functions as unidirectional transporter that mediates the outflux of a wide range of endogenous and xenobiotic compounds, thereby protecting cells against potentially toxic substances. ABCB1 has unusually wide multispecificity for numerous substrates, most of which are hydrophobic [23]. The localization of P-gp in the apical membrane of enterocytes points to its role in the efflux of possibly toxic xenobiotics out of the intestine [24]. It is noteworthy that the highest aggregation of P-gp was observed in distal part of digestive tract, especially in the colorectal region [25]. In addition, association between the $A B C B 1$ gene and the $\mathrm{Wnt} / \beta$-catenin signaling cascade was revealed; the last one is the most common dysfunction in rare hereditary forms of CRC [26]. Moreover, in vitro studies demonstrated that $A B C B 1$ expression was observed in cells with P53 mutations (P53 mutations are found in $50 \%$ of CRC cases) [27].
Considering that the main function of the $\mathrm{ABCB} 1$ product is the efflux of a number of toxic xenobiotic compounds out of cells, it can be suggested that altered P-gp function can lead to the development of intracellular toxic and chemical stress and, thereby, increase the risk of DNA damage by the products of incomplete xenobiotic metabolism (potential carcinogens), mediating the susceptibility of colon cells to tumor transformation.

In addition, it should be noted that the present study showed sex-specific effects in association of $A B C B 1$ C3435T polymorphism with the risk of CRC. In most countries of the world, the morbidity and mortality rates from CRC are considerably higher among males than among females [28]. On the other hand, several studies reported higher survival rates in females with CRC compared to males [29, 30]. First, gender can reflect different levels of environmental factors associated with occupational exposure, diet, or lifestyle. Second, sex hormones can also affect the expression of genes associated with the development of this disease. It should be noted that steroid hormones, such as progesterone, are able to modulate P-gp expression in some tissues, as evidenced by studies in mice [31]. On the other hand, SNPs can alter $A B C B 1$ expression, affecting the regulatory sequences that are targeted by steroid hormones, or can alter the ability of ABCB1 to metabolize specific substances, the consumption of which may be sex-dependent. This hypothesis is consistent with the results of Nakamura et al. [32], who suggested that serum cortisol and aldosterone levels in females could depend on $A B C B 1$ rs 1045642 polymorphism [32].

Thus, the results of the present study demonstrated that MDR1 C3435T polymorphism was associated with an increased risk of CRC in the population from Central Russia, exclusively in females. Further studies on a larger sample size, as well as replication studies, are required to confirm the role of MDR1 C3435T (rs1045642) in the pathogenesis of colorectal cancer, especially in terms of gene-gene and gene-environment interactions.

\section{FUNDING}

This study was supported by the TRC GEN+ Genetic Research Foundation of Trans Russian Co (contract no. 474).

\section{COMPLIANCE WITH ETHICAL STANDARDS}

Statement of compliance with standards of research involving humans as subjects. All procedures performed in studies involving human participants were in accordance with the ethical standards of the institutional and/or national research committee and with the 1964 Helsinki Declaration and its later amendments or comparable ethical standards.

Informed consent was obtained from all individual participants involved in the study. 
Conflicts of interest. The authors declare that they have no conflicts of interest.

\section{REFERENCES}

1. Ferlay, J., Soerjomataram, I., Dikshit, R., et al., Cancer incidence and mortality worldwide: sources, methods and major patterns in GLOBOCAN 2012, Int. J. Cancer, 2015, vol. 136, no. 5, pp. E359-E386. https://doi.org/10.1002/ijc.29210

2. Aran, V., Victorino, A.P., Thuler, L.C., and Ferreira, C.G., Colorectal cancer: epidemiology, disease mechanisms and interventions to reduce onset and mortality, Clin. Colorectal Cancer, 2016, vol. 15, no. 3, pp. 195-203. https://doi.org/10.1016/j.clcc.2016.02.008

3. Grant, D.M., Goodfellow, G.H., Sugamori, K.S., and Durette, K., Pharmacogenetics of the human arylamine N-acetyltransferases, Pharmacology, 2000, vol. 61, no. 3, pp. 204-211.

https://doi.org/10.1159/000028402

4. Probst-Hensch, N.M., Haile, R.W., Ingles, S.A., et al., Acetylation polymorphism and prevalence of colorectal adenomas, Cancer Res., 1995, vol. 55, no. 10, pp. 20172020.

5. Osian, G., Procopciuc, L., and Vlad, L., NAT2 gene polymorphism and sporadic colorectal cancer: prevalence, tumor stage and prognosis: a preliminary study in 70 patients, J. Gastrointest. Liver Dis., 2006, vol. 15, no. 4 , pp. $347-353$.

6. de Jong, M.M., Nolte, I.M., te Meerman, G.J., et al., Low-penetrance genes and their involvement in colorectal cancer susceptibility, Cancer Epidemiol. Prev. Biomarkers, 2002, vol. 11, no. 11, pp. 1332-1352.

7. Bodor, M., Kelley, E.J., and Ho, R.J., Characterization of the human MDR1 gene, AAPS J., 2005, vol. 7, no. 1, pp. E1-E5. https://doi.org/10.1208/aapsj070101

8. Kimchi-Sarfaty, C., Oh, J.M., Kim, I.W., et al., A "silent" polymorphism in the MDR1 gene changes substrate specificity, Science, 2007, vol. 315, no. 5811, pp. 525-528.

https://doi.org/10.1126/science.1135308

9. Kurzawski, M., Droździk, M., Suchy, J., et al., Polymorphism in the $\mathrm{P}$-glycoprotein drug transporter MDR1 gene in colon cancer patients, Eur. J. Clin. Pharmacol., 2005, vol. 61, nos. 5-6, pp. 389-394. https://doi.org/10.1007/s00228-005-0926-5

10. Bae, S.Y., Choi, S.K., Kim, K.R., et al., Effects of genetic polymorphisms of MDR1, FMO3 and CYP1A2 on susceptibility to colorectal cancer in Koreans, Cancer Sci., 2006, vol. 97, no. 8, pp. 774-779. https://doi.org/10.1111/j.1349-7006.2006.00241.x

11. Komoto, C., Nakamura, T., Sakaeda, T., et al., MDR1 haplotype frequencies in Japanese and Caucasian, and in Japanese patients with colorectal cancer and esophageal cancer, Drug Metab. Pharmacokinet., 2006, vol. 21 , no. 2, pp. 126-132.

12. Lee, B.I., Choi, K.Y., Lee, K.M., et al., Is C3435T polymorphism of MDR1 related to inflammatory bow- el disease or colorectal cancer in Korean? Korean J. Gastroenterol., 2006, vol. 47, no. 1, pp. 22-29.

13. Zhao, L., Li, K., Li, W., and Yang, Z., Association between the C3435T polymorphism of ABCB1/MDR1 gene (rs1045642) and colorectal cancer susceptibility, Tumor Biol., 2013, vol. 34, no. 3, pp. 1949-1957. https://doi.org/10.1007/s13277-013-0740-0

14. Jin, S.S. and Song, W.J., Association between MDR1 C3435T polymorphism and colorectal cancer risk: a meta-analysis, Medicine, 2017, vol. 96, no. 51. e9428. https://doi.org/10.1097/MD.0000000000009428

15. Moskalev, A.S., Soldatov, V.O., Vdovina, I.N., et al., The relationship between polymorphism I462V of the CYP1A1 gene and -9-154C $>$ A of the CYP1A2 gene and the colorectal cancer risk among the residents of Central Russia, Med. Genet., 2017, vol. 16, no. 3, pp. 41-45.

16. Hoffmeyer, S.O.O.H.P.J.A.I.T.I.M.U., Burk, O., Von Richter, O., et al., Functional polymorphisms of the human multidrug-resistance gene: multiple sequence variations and correlation of one allele with P-glycoprotein expression and activity in vivo, Proc. Natl. Acad. Sci. U.S.A., 2000, vol. 97, no. 7, pp. 3473-3478. https://doi.org/10.1073/pnas.050585397

17. Wu, H., Kang, H., Liu, Y., et al., Association of ABCB1 genetic polymorphisms with susceptibility to colorectal cancer and therapeutic prognosis, Pharmacogenomics, 2013, vol. 14, no. 8, pp. 897-911. https://doi.org/10.2217/pgs.13.78

18. Wang, F., Huang, Z., Zheng, K., et al., Two SNPs of ATP-binding cassette $\mathrm{B} 1$ gene on the risk and prognosis of colorectal cancer, Int. J. Clin. Exp. Pathol., 2015, vol. 8, no. 3, pp. 3083-3089.

19. Yue, A.M., Xie, Z.B., Zhao, H.F., et al., Associations of ABCB1 and XPC genetic polymorphisms with susceptibility to colorectal cancer and therapeutic prognosis in a Chinese population, Asian Pac. J. Cancer Prev., 2013, vol. 14, no. 5, pp. 3085-3091.

20. He, T., Mo, A., Zhang, K., and Liu, L., ABCB1/MDR1 gene polymorphism and colorectal cancer risk: a meta-analysis of case-control studies, Colorectal Dis., 2013, vol. 15, no. 1, pp. 12-18. https://doi.org/10.1111/j.1463-1318.2012.02919.x

21. Sheng, X., Zhang, L., Tong, N., et al., MDR1 C3435T polymorphism and cancer risk: a meta-analysis based on 39 case-control studies, Mol. Biol. Rep., 2012, vol. 39 , no. 7 , pp. $7237-7249$. https://doi.org/10.1007/s11033-012-1554-7

22. Wang, J., Wang, B., Bi, J., et al., MDR1 gene C3435T polymorphism and cancer risk: a meta-analysis of 34 case-control studies, J. Cancer Res. Clin. Oncol., 2012, vol. 138 , no. 6 , pp. 979-989. https://doi.org/10.1007/s00432-012-1171-9

23. Gatlik-Landwojtowicz, E., Äänismaa, P., and Seelig, A., Quantification and characterization of P-glycoprotein-substrate interactions, Biochemistry, 2006, vol. 45, no. 9, pp. 3020-3032. https://doi.org/10.1021/bi051380+

24. Zakeri-Milani, P. and Valizadeh, H., Intestinal transporters: enhanced absorption through P-glycoprotein- 
related drug interactions, Expert Opin. Drug Metab. Toxicol., 2014, vol. 10, no. 6, pp. 859-871. https://doi.org/10.1517/17425255.2014.905543

25. Johnstone, R.W., Ruefli, A.A., and Smyth, M.J., Multiple physiological functions for multidrug transporter P-glycoprotein? Trends Biochem. Sci., 2000, vol. 25, no. 1 , pp. $1-6$.

26. De Rosa, M., Pace, U., Rega, D., et al., Genetics, diagnosis and management of colorectal cancer, Oncol. Rep., 2015, vol. 34, no. 3, pp. 1087-1096. https://doi.org/10.3892/or.2015.4108

27. Li, X.L., Zhou, J., Chen, Z.R., and Chng, W.J., P53 mutations in colorectal cancer-molecular pathogenesis and pharmacological reactivation, World J. Gastroenterol., 2015, vol. 21, no. 1, p. 84.

28. Brenner, H., Hoffmeister, M., Arndt, V., and Haug, U., Gender differences in colorectal cancer: implications for age at initiation of screening, Br. J. Cancer, 2007, vol. 96 , no. 5 , pp. $828-831$. .

https://doi.org/10.1038/sj.bjc.6603628
29. Paulson, E.C., Wirtalla, C., Armstrong, K., and Mahmoud, N.N., Gender influences treatment and survival in colorectal cancer surgery, Dis. Colon Rectum, 2009, vol. 52, no. 12 , pp. 1982-1991. https://doi.org/10.1007/DCR.0b013e3181beb42a

30. McArdle, C.S., McMillan, D.C., and Hole, D.J., Male gender adversely affects survival following surgery for colorectal cancer, Br. J. Surg., 2003, vol. 90, no. 6, pp. 711-715. https://doi.org/10.1002/bjs.4098

31. Cui, Y.J., Cheng, X., Weaver, Y.M., and Klaassen, C.D., Tissue distribution, gender-divergent expression, ontogeny, and chemical induction of multidrug resistance transporter genes (Mdr1a, Mdr1b, Mdr2) in mice, Drug Metab. Dispos., 2009, vol. 37, no. 1, pp. 203-210. https://doi.org/10.1124/dmd.108.023721

32. Nakamura, T., Okamura, N., Yagi, M., et al., Effects of ABCB1 3435C $>$ T genotype on serum levels of cortisol and aldosterone in women with normal menstrual cycles, Genet. Mol. Res., 2009, vol. 8, no. 2, pp. 397-403.

Translated by N. Maleeva

SPELL: 1. explaned, 2. studed 